\title{
Production and characterization of crystalline ${ }^{13} \mathrm{C}_{60}$
}

\author{
I. Holleman, M.G.H. Boogaarts, P.J.M. van Bentum, G. Meijer \\ Department of Molecular and Laser Physics and High Field Magnet Laboratory, Catholic University of Nijmegen, Toernooiveld 1, \\ 6525 ED Nijmegen, The Netherlands
}

Received 23 January 1995; in final form 3 April 1995

\begin{abstract}
High-purity crystals of $99.1 \%{ }^{13} \mathrm{C}$ enriched $\mathrm{C}_{60}$ have been produced and their Raman and fluorescence spectra recorded together with those of $\mathrm{C}_{60}$ crystals containing the ${ }^{13} \mathrm{C}$ isotope in natural abundance. The relative frequency shift of the intramolecular Raman-active modes is found to be determined by the square root of the averaged mass of the $\mathrm{C}_{60}$ molecules. From the observed blue-shift of the electronic origin of the fluorescence spectrum of $\mathrm{C}_{60}$ upon ${ }^{12} \mathrm{C} \rightarrow{ }^{13} \mathrm{C}$ substitution, it is concluded that the averaged intramolecular vibrational frequency of $\mathrm{C}_{60}$ decreases slightly upon electronic excitation.
\end{abstract}

\section{Introduction}

In their first peer-reviewed Letter on the occurrence of fullerenes in laboratory produced carbon dust, Krätschmer, Fostiropoulos and Huffman used ${ }^{12} \mathrm{C} \rightarrow{ }^{13} \mathrm{C}$ isotope substitution to unambiguously assign the observed IR absorption spectra of carbon dust to large all-carbon molecules [1]. Since then, high-purity fullerenes have become available and isotope effects on various properties of fullerenes and fullerene related compounds have been studied. Isotope substitution has been used, for instance, to elucidate the mechanism of superconductivity in alkali-doped fullerenes [2]. The effect on the optical spectra of replacing ${ }^{12} \mathrm{C}$ atoms in ${ }^{12} \mathrm{C}_{60}$ by ${ }^{13} \mathrm{C}$ atoms is twofold. First, the $\mathrm{I}_{h}$ symmetry of the ${ }^{12} \mathrm{C}_{60}$ molecules is broken whereby modes that are forbidden by optical selection rules in the free icosahedral molecule become (weakly) allowed. Recent experimental results, backed by theoretical calculations, indicate that this isotopic activation of 'silent' modes does not contribute substantially to the number and intensity of the modes as observed in IR and Raman studies [3,4]. Second, the effective mass of $C_{60}$ is changed upon isotope substitution, which leads to shifts in the molecular eigenfrequencies. The latter effect is ideally studied by comparing the optical spectra of ${ }^{12} \mathrm{C}_{60}$ and ${ }^{13} \mathrm{C}_{60}$.

In the experiments reported in this Letter we have studied the shifts in the intramolecular frequencies upon isotope substitution by comparing the Raman spectrum of $\mathrm{C}_{60}$ enriched to $99.1 \%$ in the ${ }^{13} \mathrm{C}$ isotope to the Raman spectrum of $\mathrm{C}_{60}$ containing ${ }^{13} \mathrm{C}$ in natural abundance $(1.1 \%)$. In addition, dispersed fluorescence spectra of the isotopically different samples have been recorded to determine the change in the averaged vibrational frequency of $\mathrm{C}_{60}$ upon electronic excitation.

\section{Production of crystalline ${ }^{13} \mathrm{C}_{60}$}

As a starting material for the ${ }^{13} \mathrm{C}$ enriched fullerenes, $1 \mathrm{~g}$ of amorphous ${ }^{13} \mathrm{C}$ powder with a 
quoted isotope purity of $99 \%$ is used (Aldrich Chemie). This powder is sintered into six carbon rods of $15 \mathrm{~mm}$ length and $3 \mathrm{~mm}$ diameter, by pressing it under vacuum between two tantalum electrodes while sending a current through it, similar to the method reported by others [1,2].

A DC arc-discharge as experimented with by Krätschmer et al. [5] and described in more detail by Haufler et al. [6] is used to produce isotopically enriched fullerenes from the ${ }^{13} \mathrm{C}$ carbon rods. Prior to running the arc discharge, the production chamber is washed thoroughly with toluene to avoid contamination with previously produced fullerenes containing the ${ }^{13} \mathrm{C}$ isotope in natural abundance. The arc discharge is operated under a slow He flow at a total He pressure of 170 Torr, with a current of $40 \mathrm{~A}$ and a voltage drop of $25 \mathrm{~V}$ between the two identical ${ }^{13} \mathrm{C}$ carbon electrodes. As the self-made carbon rods are rather short and brittle the ${ }^{13} \mathrm{C}$ material cannot be consumed efficiently in the discharge and in total only a few hundred $\mathrm{mg}$ of soot is produced and collected. After room-temperature toluene extraction and subsequent slow evaporation of the solvent approximately $10 \mathrm{mg}$ of ${ }^{13} \mathrm{C}_{2 n}$ fullerene mixture is obtained from the soot.

Mass spectrometric characterization of the ${ }^{13} \mathrm{C}_{2 n}$ fullerene mixture is performed in a home-built laser desorption post-ionization mass spectrometer. In this spectrometer fullerenes are desorbed with a laser pulse $(532 \mathrm{~nm})$ with a low power density from a stainless steel sample substrate that is positioned close to the orifice of a pulsed $\mathrm{Kr}$ expansion. The laser desorbed fullerenes are entrained in the jet, undergo multiple collisions in the expansion region and are transported into the detection chamber. In this chamber single-photon laser ionization of the fullerenes is performed with a $F_{2}$ excimer laser ( 157 $\mathrm{nm} ; 7.90 \mathrm{eV})$, followed by mass-selective $(M / \Delta M$ $\approx 1000$ ) ion detection in a time-of-flight configuration. The relative cation intensities of the various stable fullerenes present in a sample as observed with this spectrometer closely match the real abundance of the corresponding neutral species in this sample.

In Fig. 1A a mass spectrum of a small fraction of the ${ }^{13} \mathrm{C}_{2 n}$ fullerene mixture is shown. The observed ratios between the various stable fullerenes present in the sample resemble those observed for 'normal'
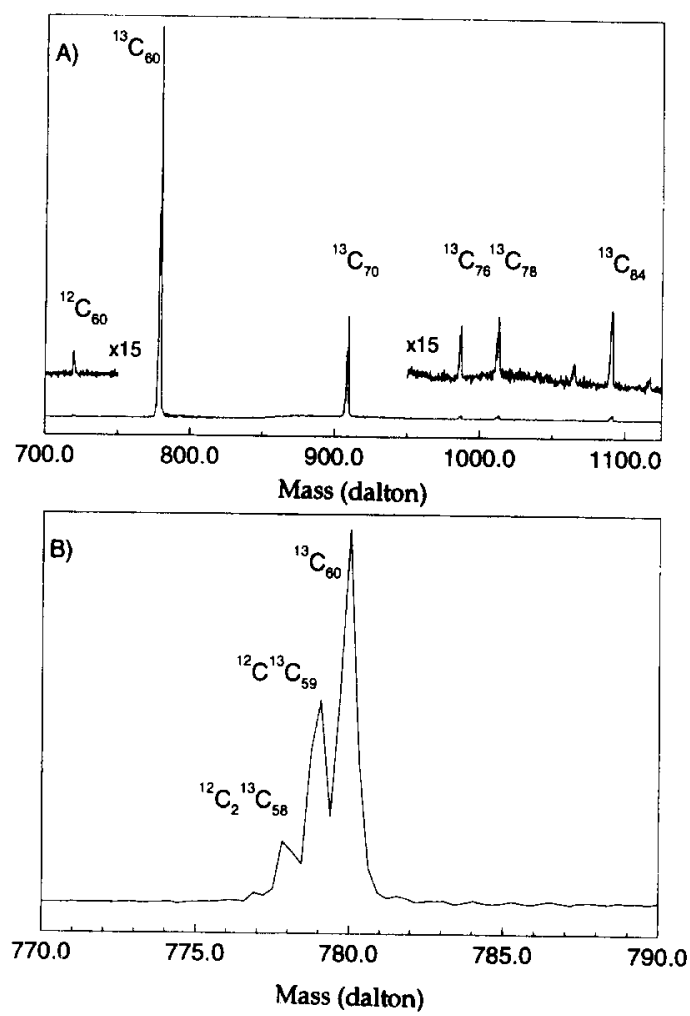

Fig. 1. Mass spectrum of ${ }^{13} \mathrm{C}$ enriched fullerene sample as measured in the laser desorption post-ionization mass spectrometer. From the observed isotope distribution a ${ }^{13} \mathrm{C}$ isotope enrichment of $99.1 \pm 0.1 \%$ is deduced.

${ }^{12} \mathrm{C}_{2 n}$ fullerene mixtures produced in the same arcdischarge source. The apparent 'contamination' of the ${ }^{13} \mathrm{C}_{2 n}$ fullerene sample with approximately $0.4 \%$ ${ }^{12} \mathrm{C}_{60}$ may well have occurred inside the mass spectrometer, and is an upper limit for the actual ${ }^{12} \mathrm{C}_{60}$ content in the sample. In Fig. $1 \mathrm{~B}$ the ${ }^{13} \mathrm{C}_{60}$ mass peak is shown on an expanded scale, showing resolved isotope structure. $\mathrm{A}^{13} \mathrm{C}$ abundance of $99.1 \pm$ $0.1 \%$ is deduced by comparing the observed isotope ratios to a binomial distribution.

High-purity ${ }^{13} \mathrm{C}_{60}$ is obtained from the fullerene mixture using an automated HPLC system. For this, $0.5 \mathrm{mg}$ of ${ }^{13} \mathrm{C}_{2 n}$ fullerene mixture dissolved in 200 $\mu l$ toluene is injected into the eluent flow $(8 \mathrm{ml} / \mathrm{min}$ of a $1: 3$ toluene:iso-octane mixture) and passed through a semi-preparative column (10 $\mathrm{mm}$ diameter, $250 \mathrm{~mm}$ length) kept at $40^{\circ} \mathrm{C}$. The fullerene separation mechanism in the column used (ChromSphere Fullerenes, Chrompack) is based on $\pi-\pi$ interac- 


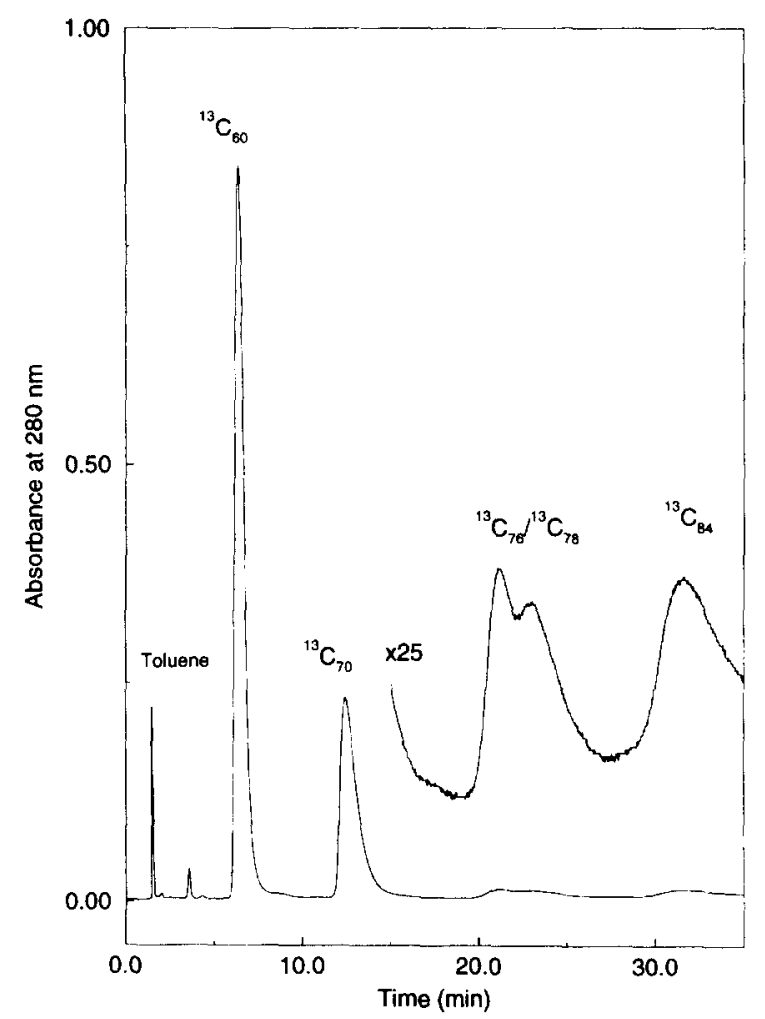

Fig. 2. HPLC chromatogram of ${ }^{13} \mathrm{C}$ enriched fullerene mixture using absorption detection at $280 \mathrm{~nm}$.

tions. After exiting the column the fullerenes are detected via their electronic absorption at $280 \mathrm{~nm}$, and are subsequently collected. In some 15 HPLC runs, lasting 45 minutes each, the fractions belonging to ${ }^{13} \mathrm{C}_{60},{ }^{13} \mathrm{C}_{70}$ and to the total amount of higher ${ }^{13} \mathrm{C}_{2 n}$ fullerenes are accumulated.

In Fig. 2 a typical chromatogram of the ${ }^{13} \mathrm{C}_{2 n}$ fullerene mixture is shown. The identification of the various peaks in the chromatogram is known from mass spectrometric analysis of the corresponding collected fractions using normal ${ }^{12} \mathrm{C}_{2 n}$ fullerenes. It is evident from the chromatogram that a single pass of the fullerene mixture through the column already yields ${ }^{13} \mathrm{C}_{60}$ of sufficient chemical purity. Mass spectrometric analysis of the collected ${ }^{13} \mathrm{C}_{60}$ fraction reveals that the chemical purity of the sample is at least $99.9 \%$, whereas a maximum of $0.4 \%$ of the sample is the 'contaminating, ${ }^{12} \mathrm{C}_{60}$ isotope. In total, approximately $6 \mathrm{mg}$ of high-purity ${ }^{13} \mathrm{C}_{60}$ powder is collected.
From the chromatographically purified ${ }^{13} \mathrm{C}_{60}$ powder crystals are grown via sublimation [7]. In short, approximately $0.5 \mathrm{mg}$ of purified powder is put in a quartz tube, and heated to $200^{\circ} \mathrm{C}$ for several hours under dynamic pumping $\left(10^{-6}\right.$ Torr) to remove residual solvent. At a temperature of around $550^{\circ} \mathrm{C}$ the ${ }^{13} \mathrm{C}_{60}$ is vapor transported into successive sections of the quartz tube to further purify the sample. Next the quartz tube is sealed off under vacuum and crystals are grown in a furnace in a temperature gradient from 620 to $600^{\circ} \mathrm{C}$. $\mathrm{A}{ }^{13} \mathrm{C}_{60}$ crystal with a diameter of approximately $0.3 \mathrm{~mm}$ is used for the optical studies.

Normal ${ }^{12} \mathrm{C}_{60}$ is known to crystallize in a fcc structure [8] although a minor fraction of the crystals (of the order of a percent) shows the hcp habit and is also microscopically pure hep with an equally high crystal quality [9]. We have only produced a limited number of ${ }^{13} \mathrm{C}_{60}$ crystals up to now and it is therefore difficult to conclude any effect of the isotope substitution on the preferred equilibrium crystal structure of high-purity $\mathrm{C}_{60}$. A detailed study on the structural properties of ${ }^{13} \mathrm{C}_{60}$ crystals is in progress.

\section{Raman and fluorescence spectra of crystalline ${ }^{13} \mathrm{C}_{60}$}

The ${ }^{13} \mathrm{C}_{60}$ crystal is mounted next to a 'normal' ${ }^{12} \mathrm{C}_{60}$ reference crystal in a He flow cryostat and evacuated to $10^{-6}$ Torr. Both crystals have been exposed to ambient air between removing them from the evacuated quartz tubes in which they are grown and evacuating the cryostat in which they are mounted for a maximum time duration of ten minutes. The optical measurements are performed at a temperature of the crystals of $10 \mathrm{~K}$. The unpolarized Raman spectra and the fluorescence spectra of the $\mathrm{C}_{60}$ crystals are recorded in backscattering geometry using a DILOR XY spectrometer. In the Raman measurements either the $514 \mathrm{~nm}$ line of an $\mathrm{Ar}$ ion laser or $760.63 \mathrm{~nm}$ radiation from an Ar ion laser pumped Ti:sapphire are used for excitation. The excitation laser is focused to a spot of approximately $15 \mu \mathrm{m}$ diameter, and typical power densities of 70 $\mathrm{W} / \mathrm{cm}^{2}$ with $514 \mathrm{~nm}$ excitation and $300 \mathrm{~W} / \mathrm{cm}^{2}$ with $760.63 \mathrm{~nm}$ excitation are employed; up to these 
power densities no (ir)reversible changes of the Raman spectra are observed at low temperatures [10]. The instrumental spectral resolution is set at 3 and 1 $\mathrm{cm}^{-1}$ for the experiments performed with 514 and $760.63 \mathrm{~nm}$ excitation, respectively. The absolute Raman frequency scale has been calibrated against both the Rayleigh scattered excitation laser light and the measured silicon Raman signal and is accurate within $1 \mathrm{~cm}^{-1}$.

In Fig. 3 the unpolarized Raman spectra measured with $514 \mathrm{~nm}$ excitation are shown for the ${ }^{13} \mathrm{C}_{60}$ crystal (Fig. 3A) together with that of the ${ }^{12} \mathrm{C}_{60}$ reference crystal (Fig. 3B). It is explicitly noted that both spectra are plotted on a different frequency scale, such that the corresponding peaks in the two
Raman spectra fall graphically exactly on top of each other to facilitate a direct comparison of the two spectra. It is evident from this figure that the overall Raman spectrum (after scaling of the frequency axis) of the two isotopically different $\mathrm{C}_{60}$ samples is similar. In both spectra, the strongest Raman peak ( $A_{g}(2)$ 'pentagonal pinch' mode) is scaled down by a factor 5. In the ${ }^{12} \mathrm{C}_{60}$ Raman spectrum the 10 expected Raman active modes for the free $\mathrm{C}_{60}$ molecule of $I_{h}$ symmetry are indicated. The arrow in the Raman spectrum of the ${ }^{13} \mathrm{C}_{60}$ crystal indicates the position of the strongest Raman mode of ${ }^{12} \mathrm{C}_{60}$, from which an upper limit for the degree of 'contamination' of the ${ }^{13} \mathrm{C}_{60}$ crystal by ${ }^{12} \mathrm{C}_{60}$ molecules of $0.5 \%$ is deduced.

Table 1

Position, width (full-width at half-maximum) and relative intensity of the most prominent lines in the Raman spectra of ${ }^{13} \mathrm{C}_{60}{ }^{\text {and }}{ }^{12} \mathrm{C}_{60}$ crystals, recorded at $10 \mathrm{~K}$ using $514 \mathrm{~nm}$ excitation light. In the last column the ratio of the line positions of ${ }^{13} \mathrm{C}_{60}$ and ${ }^{12} \mathrm{C}_{60}$ are given, with the error indicated in parentheses. The ratios denoted with an asterisk are used to determine the overall scaling factor between the Raman spectra

\begin{tabular}{|c|c|c|c|c|c|c|}
\hline \multicolumn{3}{|c|}{${ }^{13} \mathrm{C}_{60}$ crystal } & \multicolumn{3}{|c|}{${ }^{12} \mathrm{C}_{60}$ crystal } & \multirow{2}{*}{$\begin{array}{l}\text { Frequency } \\
\text { ratio }\end{array}$} \\
\hline $\begin{array}{l}\nu_{\text {Raman }} \\
\left(\mathrm{cm}^{-1}\right)\end{array}$ & $\begin{array}{l}\text { fwhm } \\
\left(\mathrm{cm}^{-1}\right)\end{array}$ & $\begin{array}{l}I \\
(\mathrm{au})\end{array}$ & $\begin{array}{l}\nu_{\text {Raman }} \\
\left(\mathrm{cm}^{-1}\right)\end{array}$ & $\begin{array}{l}\text { fwhm } \\
\left(\mathrm{cm}^{-1}\right)\end{array}$ & $\begin{array}{l}I \\
\text { (au) }\end{array}$ & \\
\hline & & & 261.8 & 4.3 & 6 & \\
\hline 255.5 & 3.7 & 14 & 266.3 & 4.2 & 10 & $0.9594(21)$ \\
\hline 261.4 & 3.8 & 8 & 271.7 & 2.9 & 15 & $0.9621(18)$ \\
\hline 411.9 & 4.1 & 24 & 428.3 & 4.3 & 21 & $0.9617(13)$ \\
\hline 416.6 & 3.7 & 20 & 433.3 & 3.5 & 16 & $0.9615(9)$ \\
\hline \multirow[t]{2}{*}{465.6} & 4.7 & 8 & 483.8 & 3.1 & 7 & $0.9624(12)$ \\
\hline & & & 489.2 & 4.4 & 14 & \\
\hline 477.2 & 3.0 & 78 & 496.1 & 2.7 & 68 & $0.9619(9)$ * \\
\hline 507.8 & 11.1 & 6 & & & & \\
\hline \multirow[t]{2}{*}{517.4} & 4.2 & 9 & & & & \\
\hline & & & 695.4 & 3.9 & 17 & \\
\hline 680.2 & 3.7 & 72 & 707.8 & 3.6 & 76 & $0.9610(8)^{*}$ \\
\hline 726.4 & 5.1 & 12 & 756.2 & 7.0 & 15 & $0.9606(11)$ \\
\hline 742.1 & 3.9 & 29 & 771.6 & 4.9 & 26 & $0.9618(8) *$ \\
\hline \multirow[t]{3}{*}{825.3} & 7.1 & 8 & 859.0 & 6.0 & 4 & $0.9608(11)$ \\
\hline & & & 973.1 & 13.6 & 7 & \\
\hline & & & 1068.4 & 3.8 & 10 & \\
\hline 1036.7 & 3.1 & 21 & 1078.7 & 3.5 & 26 & $0.9611(5)^{*}$ \\
\hline 1056.8 & 4.8 & 14 & 1099.4 & 3.3 & 12 & $0.9613(6)$ \\
\hline 1094.5 & 7.3 & 7 & 1137.8 & 12.1 & 9 & $0.9619(12)$ \\
\hline 1361.4 & 3.0 & 11 & 1416.8 & 3.9 & 15 & $0.9609(4)$ \\
\hline 1369.5 & 4.5 & 41 & 1424.0 & 4.8 & 30 & $0.9617(5)$ * \\
\hline 1411.8 & 3.3 & 1000 & 1467.1 & 3.2 & 1000 & $0.9623(3)^{*}$ \\
\hline 1423.2 & 4.8 & 43 & 1478.0 & 7.5 & 40 & $0.9629(7)$ \\
\hline 1443.8 & 8.6 & 5 & 1497.8 & 3.5 & 9 & $0.9639(7)$ \\
\hline 1514.2 & 5.8 & 27 & 1574.6 & 5.0 & 23 & $0.9616(5)^{*}$ \\
\hline 1626.9 & 20.0 & 10 & 1689.5 & 12.6 & 9 & $0.9629(14)$ \\
\hline
\end{tabular}



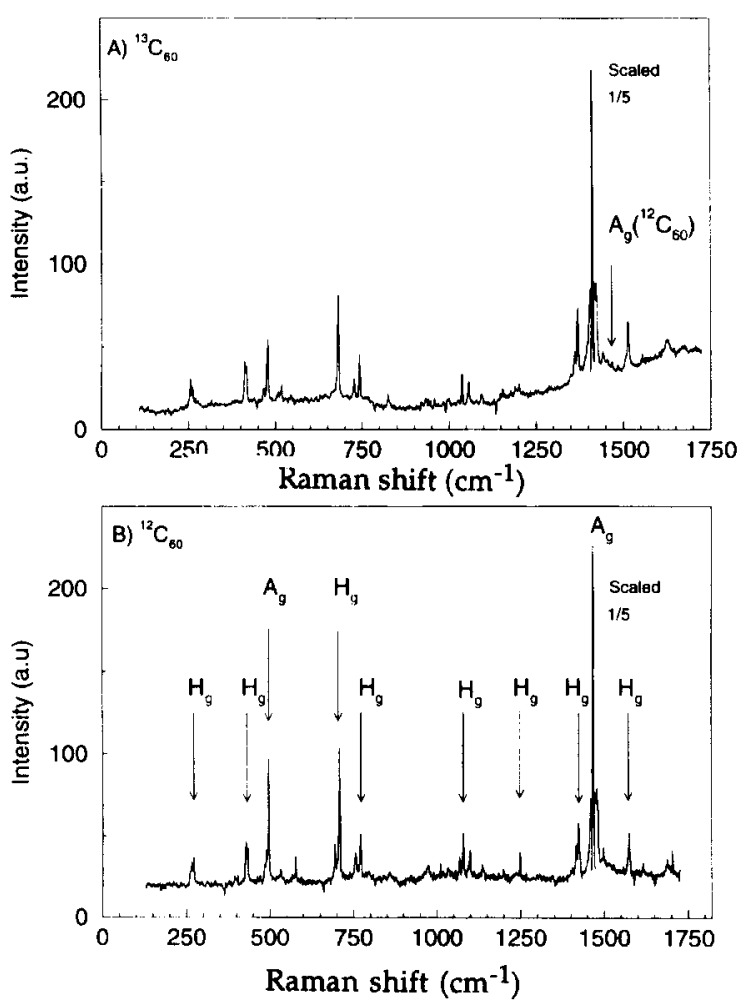

Fig. 3. Unpolarized Raman spectra of ${ }^{13} \mathrm{C}_{60}$ and ${ }^{12} \mathrm{C}_{60}$ crystals recorded at $10 \mathrm{~K}$ using $514 \mathrm{~nm}$ excitation light. The spectra are plotted on a different frequency scale (factor 0.9616 ) to facilitate comparison. In the ${ }^{12} \mathrm{C}_{60}$ Raman spectrum the ten expected Raman active modes for the free $\mathrm{C}_{60}$ molecule of $\mathrm{I}_{\mathrm{h}}$ symmetry are indicated. The strongest Raman peak is scaled down by a factor 5 in both spectra and the position of this peak for ${ }^{12} \mathrm{C}_{60}$ is also indicated in the Raman spectrum of ${ }^{13} \mathrm{C}_{60}$.
In Table 1 the frequencies of the observed Raman lines for both crystals are given, together with the observed linewidths and the relative intensities. Apart from the 10 modes that are allowed for the free $C_{60}$ molecule, additional modes are observed in both crystals. These modes have been attributed to solid state effects [11] i.e. to activation of silent modes due to the reduced symmetry of $\mathrm{C}_{60}$ in the crystalline lattice. The similarity of the Raman spectra of the isotopically different species implies, therefore, that the crystalline environment is similar in both cases. It is noted that at least some of the additional lines can alternatively be interpreted as combination modes [12]. In the last column of Table 1 the ratios of the observed Raman frequencies for ${ }^{13} \mathrm{C}_{60}$ relative to the observed frequencies for ${ }^{12} \mathrm{C}_{60}$ are given. Only well-isolated and relatively strong Raman lines in both spectra (whose ratio is indicated with an asterisk) have been used to determine the overall scaling factor between the two spectra as $0.9616 \pm 0.0005$, and this scaling factor has been used in the graphic presentation of the Raman spectra in Fig. 3.

Similar Raman measurements have been performed using $760.63 \mathrm{~nm}$ excitation. The results obtained in this study are summarized in Table 2. It is known that the overall intensity distribution in the Raman spectra is rather different in such a non-resonant Raman scheme [11,13]. In addition, it is noted that the spectral response of our spectrometer declines rapidly for wavelengths longer then $760 \mathrm{~nm}$. The relative intensity measurements presented in

Table 2

Position, width (full-width at half-maximum), relative intensity and frequency ratio of the most prominent lines in the Raman spectra of ${ }^{13} \mathrm{C}_{60}$ and ${ }^{12} \mathrm{C}_{60}$ crystals, recorded at $10 \mathrm{~K}$ using $760.63 \mathrm{~nm}$ excitation light

\begin{tabular}{|c|c|c|c|c|c|c|}
\hline \multicolumn{3}{|c|}{${ }^{13} C_{60}$ crystal } & \multicolumn{3}{|c|}{${ }^{12} \mathrm{C}_{60}$ crystal } & \multirow{2}{*}{$\begin{array}{l}\text { Frequency } \\
\text { ratio }\end{array}$} \\
\hline $\begin{array}{l}\nu_{\text {Raman }} \\
\left(\mathrm{cm}^{-1}\right)\end{array}$ & $\begin{array}{l}\text { fwhm } \\
\left(\mathrm{cm}^{-1}\right)\end{array}$ & $\begin{array}{l}I \\
\text { (au) }\end{array}$ & $\begin{array}{l}\nu_{\text {Raman }} \\
\left(\mathrm{cm}^{-1}\right)\end{array}$ & $\begin{array}{l}\text { fwhm } \\
\left(\mathrm{cm}^{-1}\right)\end{array}$ & $\begin{array}{l}I \\
\text { (au) }\end{array}$ & \\
\hline 255.7 & 0.9 & 186 & 265.9 & 1.2 & 216 & $0.9616(6)^{*}$ \\
\hline 261.5 & 1.3 & 1000 & 271.9 & 1.1 & 1000 & $0.9618(6)^{*}$ \\
\hline 412.2 & 9.5 & 62 & 428.6 & 6.3 & 47 & $0.9617(26)$ \\
\hline 416.6 & 1.4 & 101 & 433.4 & 0.9 & 157 & $0.9612(4) *$ \\
\hline 465.5 & 2.0 & 76 & 483.7 & 4.1 & 73 & $0.9624(9)^{*}$ \\
\hline 476.9 & 1.1 & 884 & 496.1 & 1.0 & 735 & $0.9613(3)^{*}$ \\
\hline 680.4 & 3.1 & 58 & 707.8 & 2.3 & 40 & $0.9613(6)^{*}$ \\
\hline 743.1 & 1.8 & 127 & 772.4 & 2.1 & 114 & $0.9621(4)$ * \\
\hline 1412.4 & 1.4 & 316 & 1467.8 & 1.9 & 216 & $0.9623(2)^{*}$ \\
\hline
\end{tabular}


Table 2 have not been corrected for the spectral response of the spectrometer as it plays only a minor role in the comparison of the Raman spectra of the isotopically different crystals. From these measurements the overall scaling factor between the Raman frequencies for the ${ }^{13} \mathrm{C}_{60}$ crystal relative to the ${ }^{12} \mathrm{C}_{60}$ crystal is found to be $0.9618 \pm 0.0005$.

The combined result of these measurements yields an observed frequency ratio between the intramolecular modes of $99.1 \pm 0.1 \%{ }^{13} \mathrm{C}$ enriched $\mathrm{C}_{60}$ relative to $\mathrm{C}_{60}$ containing the ${ }^{13} \mathrm{C}$ isotope in natural abundance $(1.1 \%)$ of $0.9617 \pm 0.0004$. This ratio is significantly different from $\sqrt{12 / 13}=0.9608$, but is within the error limit identical to $\sqrt{12.011 / 12.991}=0.9615$. This result implies that the observed Raman lines are found at the position determined by the averaged mass of the $C_{60}$ molecules and that the Raman spectrum of, for instance, ${ }^{12} \mathrm{C}_{59}{ }^{13} \mathrm{C}$ is nearly identical to that of ${ }^{12} \mathrm{C}_{60}$ and is displaced within the effective linewidth of the modes.

Recently, a detailed study on the fluorescence of crystalline $\mathrm{C}_{60}$ (natural ${ }^{13} \mathrm{C}$ abundance) at low temperature was completed in which it was demonstrated that the most intense spectral feature in the fluorescence spectrum of crystalline $\mathrm{C}_{60}$ was the electronic origin at $13631 \mathrm{~cm}^{-1}(733.6 \mathrm{~nm})$ [14]. Using excitation with $514 \mathrm{~nm}$ at low power densities $\left(\leqslant 10 \mathrm{~W} / \mathrm{cm}^{2}\right)$ the dispersed fluorescence spectra of both crystals have been recorded at $10 \mathrm{~K}$ in the spectral region around this origin. In Fig. 4 the measured fluorescence spectra, not corrected for the spectral response of the spectrometer, are shown for the two isotopically different samples. Although the origins in both fluorescence spectra nearly coincide, there is a small blue-shift of $13 \pm 7 \mathrm{~cm}^{-1}$ of the ${ }^{13} \mathrm{C}_{60}$ origin relative to the ${ }^{12} \mathrm{C}_{60}$ origin. The width of the fluorescence peaks is determined as 240 and $180 \mathrm{~cm}^{-1}$ for ${ }^{13} \mathrm{C}_{60}$ and ${ }^{12} \mathrm{C}_{60}$, respectively.

The observed blue-shift of this peak is a measure of the change of the averaged intramolecular vibrational frequency of $\mathrm{C}_{60}$ upon electronic excitation, which can be seen as follows. Both the electronic potential energy surface of the ground state and the electronically excited state of $\mathrm{C}_{60}$ will not change upon isotope substitution. In addition, the zero-point vibrational energy contributions in both electronic states will scale with the same factor 0.9617 upon
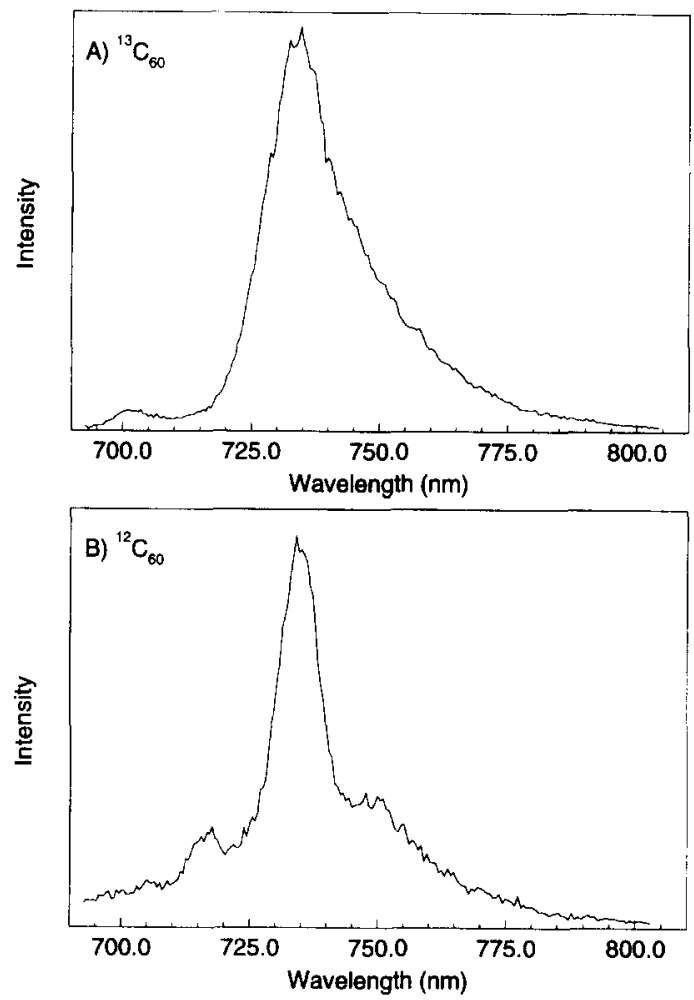

Fig. 4. Dispersed fluorescence spectra of ${ }^{13} \mathrm{C}_{60}$ and ${ }^{12} \mathrm{C}_{60}$ crystals recorded at $10 \mathrm{~K}$ using $514 \mathrm{~nm}$ excitation light.

${ }^{12} \mathrm{C} \rightarrow{ }^{13} \mathrm{C}$ isotope substitution. Therefore, any observed shift in the origin of the electronic transition must originate from differences in zero-point vibrational energy in the states coupled in the transition. The observed blue-shift of $13 \pm 7 \mathrm{~cm}^{-1}$ implies a lower zero-point vibrational energy contribution in the electronically excited state of $\mathrm{C}_{60}$ than in the electronic ground state, by an amount given by $(13 \pm 7) /(1-0.9617) \mathrm{cm}^{-1}=340 \pm 180 \mathrm{~cm}^{-1}$ for ${ }^{12} \mathrm{C}_{60}$. Relative to the total zero-point vibrational energy contribution, which in the ground state of ${ }^{12} \mathrm{C}_{60}$ is around $82640 \mathrm{~cm}^{-1}$ [12], this implies a decrease in averaged vibrational energy of $0.4 \pm$ $0.2 \%$ upon electronic excitation of $\mathrm{C}_{60}$. Interestingly, this decrease is of the same order of magnitude as that found by exciting $\mathrm{C}_{60}$ molecules to the triplet manifold [10].

It is noted that the near coincidence of the peaks in the fluorescence spectra of the isotopically different crystals supports the interpretation that the fluorescence band around $733.6 \mathrm{~nm}$, the strongest band 
in the fluorescence spectrum of $\mathrm{C}_{60}$, is indeed the electronic origin band [14]. This fluorescence peak has alternatively been interpreted as a $t_{1 u}$ false origin band, with the true electronic origin lying 1430 $\mathrm{cm}^{-1}$ higher in energy [15]. If this latter interpretation were correct, a blue-shift of the $733.6 \mathrm{~nm}$ fluorescence peak of $55 \mathrm{~cm}^{-1}$ would result from the change of the frequency of the $t_{1 u}$ mode upon ${ }^{12} \mathrm{C}$ $\rightarrow{ }^{13} \mathrm{C}$ substitution. This would only be consistent with the observed blue-shift of $13 \pm 7 \mathrm{~cm}^{-1}$, if the averaged vibrational frequency in $\mathrm{C}_{60}$ were to increase by $1.3 \pm 0.2 \%$ upon electronic excitation, which is highly unlikely.

\section{Conclusions}

High-purity crystals of $99.1 \%{ }^{13} \mathrm{C}$ enriched $\mathrm{C}_{60}$ have been produced and the optical properties of these crystals have been compared to those of "normal' ( ${ }^{13} \mathrm{C}$ isotope abundance $1.1 \%$ ) $\mathrm{C}_{60}$ crystals. The Raman spectra of the isotopically different samples are similar overall, and the frequency scaling factor due to the different masses of the isotopes is found to be $0.9617 \pm 0.0004$. This implies that the frequency position of each Raman mode is governed by the weighed average of the corresponding frequency positions of the various isotopes that are still present in the isotopically enriched crystals. The fluorescence spectra of both crystals are also similar and the electronic origins nearly coincide. The small blueshift that is observed in the origin of the first electronically allowed transition of $\mathrm{C}_{60}$ upon ${ }^{12} \mathrm{C} \rightarrow{ }^{13} \mathrm{C}$ isotope substitution implies a $0.4 \pm 0.2 \%$ decrease in the averaged vibrational frequency of $\mathrm{C}_{60}$ upon electronic excitation.

\section{Acknowledgement}

We acknowledge the help of P.H.M. van Loosdrecht and D.S. Bethune in preparing the ${ }^{13} \mathrm{C}$ carbon rods and the help of M.A. Verheijen in the production of the crystals. This work is part of the research program of the 'Stichting voor Fundamenteel Onderzoek der Materie (FOM)', and is financially supported by the 'Nederlandse Organisatie voor Wetenschappelijk Onderzoek (NWO)' via a PIONIERgrant.

\section{References}

[1] W. Krätschmer, K. Fostiropoulos and D.R. Huffman, Chem. Phys. Letters 170 (1990) 167.

[2] Ch.Ch. Chen and Ch.M. Lieber, J. Am. Chem. Soc. 114 (1992) 3141.

[3] M.C. Martin, J. Fabian, J. Godard, P. Bernier, J.M. Lambert and L. Mihaly, Phys. Rev. B, in press.

[4] A. Rosenberg and C. Kendziora, Phys. Rev. B, in press.

[5] W. Krätschmer, L.D. Lamb, K. Fostiropoulos and D.R. Huffman, Nature 347 (1990) 354.

[6] R.E. Haufler, Y. Chai, L.P.F. Chibante, J. Conceicao, Ch. Jin, L.-Sh. Wang, Sh. Maruyama and R.E. Smalley, Mat. Res. Soc. Symp. Proc. 206 (1991) 627.

[7] M.A. Verheijen, W.J.P. van Enckevort and G. Meijer, Chem. Phys. Letters 216 (1993) 72.

[8] R.M. Fleming, T. Siegrist, P.M. Marsh, B. Hessen, A.R. Kortan, D.W. Murphy, R.C. Haddon, R. Tycko, G. Dabbagh, A.M. Mujsce, M.L. Kaplan and S.M. Zahurak, Mat. Res. Soc. Symp. Proc. 206 (1991) 691.

[9] J.L. de Boer, S. van Smaalen, V. Petricek, M. Dusek, M.A. Verheijen and G. Meijer, Chem. Phys. Letters 219 (1994) 469.

[10] P.H.M. van Loosdrecht, P.J.M. van Bentum and G. Meijer, Chem. Phys. Letters 205 (1993) 191.

[11] P.H.M. van Loosdrecht, P.J.M. van Bentum, M.A. Verheijen and G. Meijer, Chem. Phys. Letters 198 (1992) 587.

[12] Zh.-H. Dong, P. Zhou, J.M. Holden, P.C. Eklund, M.S. Dresselhaus and G. Dresselhaus, Phys. Rev. B 48 (1993) 2862.

[13] B. Chase and P.J. Fagan, J. Am. Chem. Soc. 114 (1992) 2252.

[14] D.J. van den Heuvel, I.Y. Chan, E.J.J. Groenen, M. Matsushita, J. Schmidt and G. Meijer, Chem. Phys. Letters 233 (1995) 284.

[15] W. Guss, J. Feldmann, E.O. Göbel, C. Taliani, H. Mohn, W. Müller, P. Häussler and H.-U. ter Meer, Phys. Rev. Letters 72 (1994) 2644. 